\title{
Fast Handoff Scheme for VoIP over WLANs
}

\author{
Jun Xiao, Feng Liu \\ School of Computer and Information Technology, Beijing Jiaotong University, Beijing, China \\ $\{09112070$, fliu $\} @$ bjtu.edu.cn
}

\begin{abstract}
IEEE 802.11 wireless local area networks (WLAN) have been widely deployed, and many applications are implemented on WLAN. As one of the interesting wireless services, wireless voice over internet protocol (VoIP) can provide low-cost real-time voice service to mobile users. But the IEEE 802.11 wireless network cannot satisfy the QoS of VoIP. The delay during handoff process can last up to 1 second. Applications with delay restraint like VoIP will drop the call when the delay is more than 50 ms. Therefore this paper analyzed the delay made by handoff process, and proposed a fast handoff scheme. This fast handoff scheme is based on selective scanning and caching. It can reduce the handoff delay to about $30 \mathrm{~ms}$ and avoid necessary handoff. Simulations are implemented and the results show the proposed fast handoff scheme is useful and economical.
\end{abstract}

Keywords-IEEE 802.11; handoff; VoIP; QoS; scanning

\section{INTRODUCTION}

In recent years, IEEE 802.11 wireless local area networks (WLAN) have been widely deployed, and provide wireless internet connection in many places such as home, office rooms, and commercial centers, not only in buildings but also in parks, streets and vehicles. And many mobile devices such as laptops, tablet computers, and cell phones have integrated with IEEE 802.11 equipment. As wireless networks becoming more accessible, many interesting wireless applications and services are provided to users. As one of the interesting wireless services, wireless voice over internet protocol (VoIP) can provide low-cost real-time voice service to mobile users.

IEEE 802.11 standard [1] have two operating modes, infrastructure and ad-hoc mode. The infrastructure mode distinguished by the use of an access point is the most common form of deployments. In infrastructure networks, APs are used in all communications, including the communications between mobile stations in the same BSS. This mode can reduce configuration and energy. But it limits the use area of WLAN, since APs have a small range. Once a STA moves out of the range of current AP in a BSS, it needs a new AP and associates with it. This process is called layer 2 handoff or MAC layer handoff which is shown in Fig. 1. When a STA is moving away from the current AP, the signal strength and the signal-to-noise ratio (SNR) of the signal from the AP decrease. Then the STA breaks its association with the current AP, and scans a new AP to associate with. Essentially, during the handoff process, the STA's communication is suspended and all incoming packets will be dropped. Depending on different handoff strategy, the delay during handoff process can last up to 1 second.
Applications with delay restraint like VoIP will drop the call when the delay is more than $50 \mathrm{~ms}$ [2]. So the handoff strategy must be improved for the real-time applications.

Therefore this paper analyzed the handoff process, and found out the time-consuming step in the handoff process. Then a fast handoff scheme is proposed. It can reduce the delay in MAC layer handoff no more than $40 \mathrm{~ms}$ which is agree with the satisfaction of VoIP. This fast handoff scheme can be used in existing mobile devices, while the existing APs do not need changed. So this scheme is useful and economical.

The rest of this paper is organized as follows. Section II presents the IEEE 802.11 background and the related work, while the analysis of handoff process is presented in this section. Section III provides an operation detail of the fast handoff scheme. In section IV, some simulations are implemented and the results are discussed. Section V concludes this paper.

\section{BACKGROUND AND RELATED WORK}

The basic handoff process consists of three phases: scanning, authentication and re-association.

Scanning can be accomplished either in passive or active mode according to the IEEE 802.11 standard.

In passive mode, the client listens to the wireless medium for beacon frames in each channel, which provide a combination of timing and control information to clients. Using the information and the signal strength of beacon frames, the client selects an AP to join. But clients need to stay on each channel at least for one beacon interval to listen to beacons from all APs on this channel.

In active scanning mode, the client to broadcast probe requests evoking probe responses from APs actively without waiting for any beacon frames. The active scanning process is as following steps which are depicted in Fig. 2. Firstly The STA in the scanning phase broadcasts a probe request frame to APs in a channel and sets on a probe timer. Then listening for probe responses, if no response has been received by

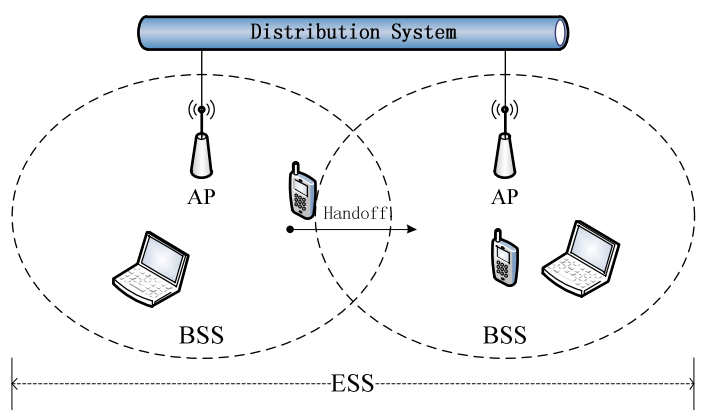

Figure 1. A typical handoff scenario. 
minChannelTime, the STA will probe the next channel. If one or more responses are received by minChannelTime, the STA will continue accepting probe responses until maxChannelTime. Next, the STA moves to the next channel and repeats the above steps. After all channels have been scanned, all information received from probe responses is processed so that the STA can select a best AP to join next.

Authentication is a process in which the AP will decide whether to accept the identity of the STA. In this process the STA sends an authentication request frame carrying the identity information to the AP. Then the AP responds with an authentication response frame, indicating acceptance or rejection for the STA.

Association process is after the successful authentication, the STA sends a re-association request to the new AP, which will then send a re-association response containing acceptance or rejection to the STA.

In the three phases, the probe delay is the dominating component which accounts for more than $90 \%$ of the overall handoff delay [3]. For this reason, many researches on fast (or seamless) handoff in IEEE 802.11 are focused on reducing the scanning time as discussed later.

Active scanning is much faster than passive scanning and

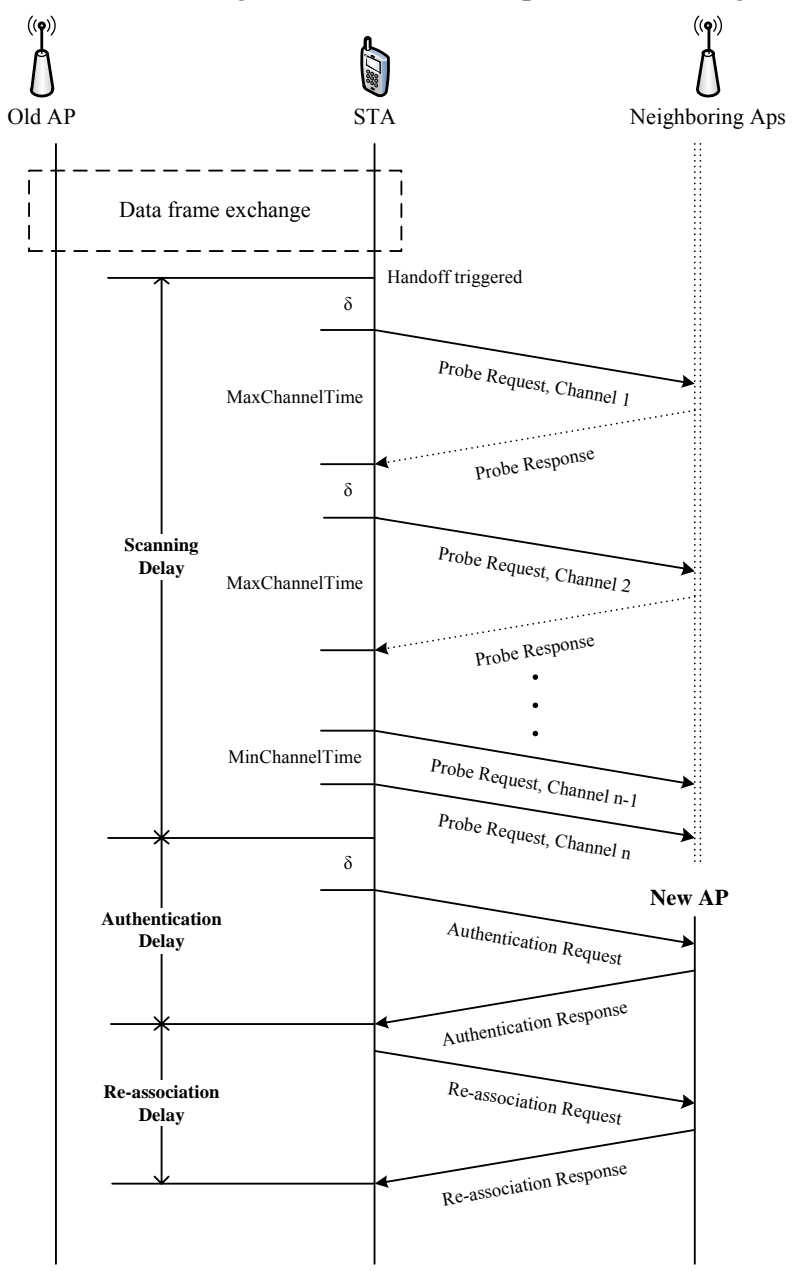

Figure 2. IEEE 802.11 MAC layer handoff process with active scanning. is more widely using in practice for handoff situations. The active scanning delay $T_{A S}$ is given by

$$
\left\{\begin{array}{l}
N \times \text { minChannelTime }+N \times \delta \leqslant T_{A S} \\
T_{A S} \leqslant N \times \text { maxChannelTime }+N \times \delta
\end{array}\right.
$$

where $\delta$ is the time taken to switch the radio from one channel to another, $N$ is the number of channels in the IEEE 802.11 spectrum, minChannelTime and maxChannelTime are set by STA for waiting for response frame. $\delta$ is varying from 5 to $10 \mathrm{~ms}$ depending on the vendor implementation. The minChannelTime and maxChannelTime are tunable parameters and various studies ([3] and [4]) have suggested them. If minChannelTime is $5 \mathrm{~ms}$ and maxChannelTime is 11 $\mathrm{ms}$, the active scanning varies from $110 \mathrm{~ms}$ to $176 \mathrm{~ms}$ in IEEE $802.11 \mathrm{~b}$ networks. So active scanning is much faster than passive scanning and is more widely using in practice for handoff situations.

Once scanning is over and the best AP has been identified, the STA will start the authentication process and re-association process. Adding the delay of these two phases, the aggregate handoff delay is given by

$$
T_{\text {handoff }}=T_{\text {Scaning }}+T_{\text {Authentication }}+T_{\text {Re-association }} .
$$

So the handoff delay is about $200 \mathrm{~ms}$ on average in an IEEE $802.11 \mathrm{~b}$ networks using 11 channels and active scanning. Though active scanning is much faster, it cannot satisfy the real-time applications whose delay requirement is no more than $50 \mathrm{~ms}$. So many researches discussed the potential optimizations of the scanning delay.

Calculating suitable values of minChannelTime and maxChannelTime for handoff is proposed first. This approach did not alter the original IEEE 802.11 handoff strategy. MinChannelTime represents the minimum duration a STA must wait after sending out a probe request and before deciding that the channel is empty. If it is too small, the STA will not find any AP in this channel. If it is too long, the STA will waste time in empty channel. In [3] and [4], the suitable MinChannelTime value is from $1 \mathrm{~ms}$ to $7 \mathrm{~ms}$. MaxChannelTime is the maximum duration a STA has to wait for probe responses from APs in this channel. This value should ensure that probe responses from most or all of the APs are collected by the STA before moving to the next channel. This value is depends on the AP density in each channel. This value should be naturally minimized to reduce the scanning phase, but it may result in candidate APs being missed. In [3] and [4], the suitable MaxChannelTime is around $11 \mathrm{~ms}$ which can suffice in practice to capture most or all probe responses. This approach can reduce the delay, but in AP dense networks the delay is also too high to support the real-time applications.

In [5] and [6], they proposed a selective scanning approach which scanning some channels rather than all channels. APs operate on orthogonal channels to minimize adjacent channel interference (e.g. only 3 such channels for IEEE 802.11 b). Each BSS is typically surrounded by a few 
APs operating on a subset of the total number of channels. Clearly, there is no need to scan all $802.11 \mathrm{~b}$ channels, as some channels would just be devoid of APs. Based on previous scans, STAs store the information about the best AP for each neighboring channel. This approach scans fewer channels than the traditional approach, so it reduces much more time on scanning. But it depends largely on the WLAN topology. If the topology is changed, this approach will be invalid.

Multiple radios are proposed in [7]. In this approach a STA needs at least two radios, one of which is reserved for scanning, and the others are used for normal operation. The STA can exchange data frames with a serving AP while it performs scanning. So this approach can realize a seamless handoff. However, since typical commercial STAs, such as cell phone, are not equipped with multiple radios. Anymore, only for scanning, using multiple radios will waste more energy.

Another approach is using GPS for movement detection, which can exactly compute STAs's site and pick out the suitable next APs for them. In [8], an efficient GPS-based handoff scheme is designed for vehicular networks. AP selection scheme can find the best AP within a vehicle's transmission range for a handoff, which is located on the vehicles potential path so that it can maintain a long and stable connection with the vehicle. This approach significantly reduces the time and the number of handoffs using GPS in vehicular networks. However GPS is not suitable for indoors networks.

A fast handoff scheme will be proposed in this paper to reduce the handoff delay. This scheme will be useful and economical.

\section{FAST HANDOFF SCHEME}

Since the analysis has been presented in section II, the scanning phase caused main delay in handoff. So the proposed fast handoff scheme is mainly work on the improvement of scanning phase. This scheme is based on selective scanning and caching. It consists of scanning trigger, channel scanner, handoff trigger, and caching.

\section{A. Scanning Trigger}

The scanning trigger is different from the handoff trigger. Scanning trigger is more sensitive than handoff trigger. When the STA does not transmit real-time package, scanning will do before the handoff is implemented. In this paper, RSSI and an accelerometer are used as scanning trigger. A STA scans channels only when the RSSI from its current AP is below a specified threshold and it is moving.

RSSI is a reasonable handoff trigger. Clients implement handoff mainly for they are moving out of the range of current AP. The distance between the client and its current AP directly represents the need of handoff for the moving client. RSSI is inversely proportional to the distance in Fig. 3. The client is moving from AP1 to AP2. When the client is far away from AP1, the RSSI of AP1 is decreasing gradually. Conversely, when the client is close with AP2, the RSSI of AP2 is increasing gradually. So RSSI is a suitable trigger reflected the movement of clients.

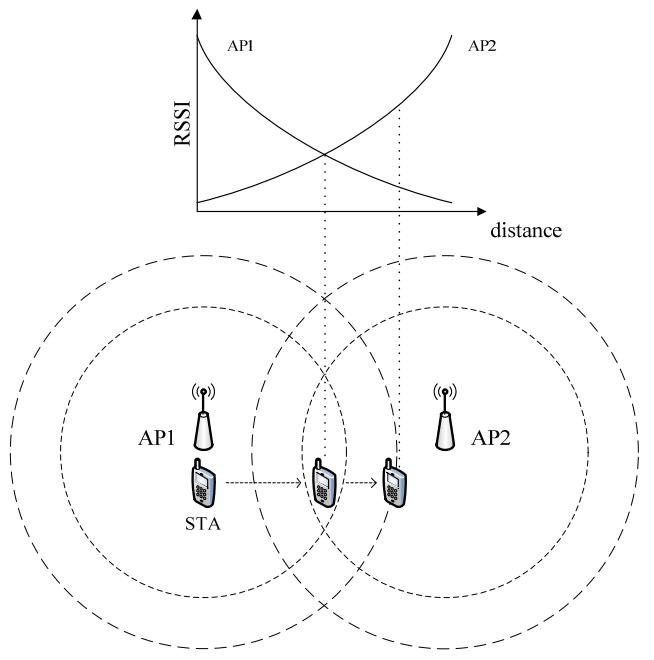

Figure 3. RSSI of a STA which is moving from AP1 to AP2.

The accelerometer is a motion sensor which is already deployed in many commercial smartphones. It is accurate and energy efficient [9]. A SAT needs a handoff when it is moving from a current AP to another AP. So an accelerometer can be used to detect the motion status of a STA, only when it is moving and the RSSI is below the threshold, the STA implements a handoff.

\section{B. Channel Scanner}

Wireless clients learn about available APs by scanning other IEEE 802.11 channels for available APs on the same WLAN. Scanning other IEEE 802.11 channels can be performed actively or passively. This scheme used the active scanning.

Active scanning switches to a channel, broadcasts a probe request, and then waits to hear any probe responses from APs on that channel. The probe-request frames used in an active scan are two types. First one is directed probe, in which the STA sends a probe request with a specific destination SSID, and only APs with a matching SSID will reply with a probe response. Another is broadcast probe, in which the client sends a broadcast probe request, and all APs receiving the probe-request will respond with a proberesponse for each SSID they support.

Directed probe is used when there are next APs in caching. The STA will probe those APs in caching in order from high connection count to low count. After a handoff, the connection count of the AP, which next associates with the STA, will plus one in caching.

Selective scanning is often used. In IEEE $802.11 \mathrm{~b}$ networks, only three channels (channel 1, 6 and 11) are not overlap in Fig. 4. Therefore, most of APs in a WLAN are configured with these non-overlap channels to avoid channel interference. Scanning these channels firstly can have more chance of find out next AP and save more time than full scanning. When a next AP is not found in these channels, the STA will scan the rest channels. 


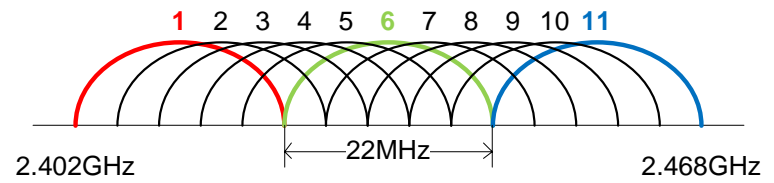

Figure 5. Channel frequency distribution in IEEE

\section{Handoff Trigger}

The handoff trigger in IEEE 802.11 standard is used for a STA to decide making a handoff in current connection. Handoff trigger is an important component in handoff algorithm. If the handoff trigger is too sluggish, clients will suffer an unaccepted latency because they cannot connect to new APs immediately. While if the handoff trigger is too sensitive, the client will make unnecessary handoff so frequently that it will cause congestion in a busy network and consume more power.

The handoff threshold is lower than scanning threshold. If the scanning is finished and the best next AP is found out, the STA will implement handoff directly without scanning. Otherwise the STA will wait for scanning.

\section{Caching}

Caching is used for recording the APs which are scanned and associated by the STA, and offers useful information for channel scanning.

When a scanning is finished, the scanned APs will be put in caching table in Table. 1 . The current AP is the AP which is current connecting with the STA. And the scanned AP is the AP which is scanned by the STA when RSSI of the moving STA is below the scanning threshold with current AP. For example, when RSSI of the moving STA is below the scanning threshold with AP1, the STA scans and finds AP2, AP3 and AP4. So there are three entries recorded in caching table. The AP information in every entry includes MAC address and SSID of this AP.

When a handoff is completed, the connection count of the new associated AP in caching table will increase one. For example, when the STA makes a handoff from AP1 to AP2, the connection count in the entry about AP1 and AP2 will plus one.

When a new channel scanning is triggered, the STA will search caching table, find out entries about the current AP, order connection count from high count to low count, and

TABLE I. DELAY PARAMETERS USED IN SimULATIONS

\begin{tabular}{|c|c|}
\hline Parameters & Delay \\
\hline MaxChannelTime & $200 \mathrm{~ms}$ \\
\hline MinChannelTime & $20 \mathrm{~ms}$ \\
\hline Channel switching time & $11.4 \mathrm{~ms}$ \\
\hline Authentication delay & $6 \mathrm{~ms}$ \\
\hline Re-association delay & $4 \mathrm{~ms}$ \\
\hline
\end{tabular}

scan these AP orderly by directed probe. For example, when the STA reaches the scanning threshold with current AP1, it will find out three entries about AP1 in caching table. And it will scan as this sequence, AP2 $\rightarrow$ AP3 $\rightarrow$ AP4, until a suitable AP replies with probe response frame.

\section{SimUlATIONS AND ANALYSIS}

Simulations are implemented on OPNET Modeler 14.5 which is a commercial network simulation tool.

An IEEE 802.11b wireless network is deployed in Fig.5. There are ten APs which are non-overlap, and a STA which can move among the 36 blocks. The trajectories of the STA are created by a computer procedure. There are three trajectory types, cycle, return and random. In each trajectory type, the STA will go through 50000 blocks (total length is $12500 \mathrm{~km}$ ) by $5 \mathrm{~km} / \mathrm{h}$ about 2500 hours. To make comparisons, full scanning and non-overlap scanning are also implemented. The delay parameters used in simulations is showed in Table. 2.

The average delay is showed in Fig.6. The fast handoff scheme can reduce the delay vastly. The delay of fast handoff scheme is about $30 \mathrm{~ms}$, which can satisfy the VoIP requirement. And in Fig.7, the number of handoff also decreases, especially in cycle and return trajectories. Avoiding the necessary handoffs, the STA can save power energy. Therefore the proposed fast handoff scheme is useful and economical.

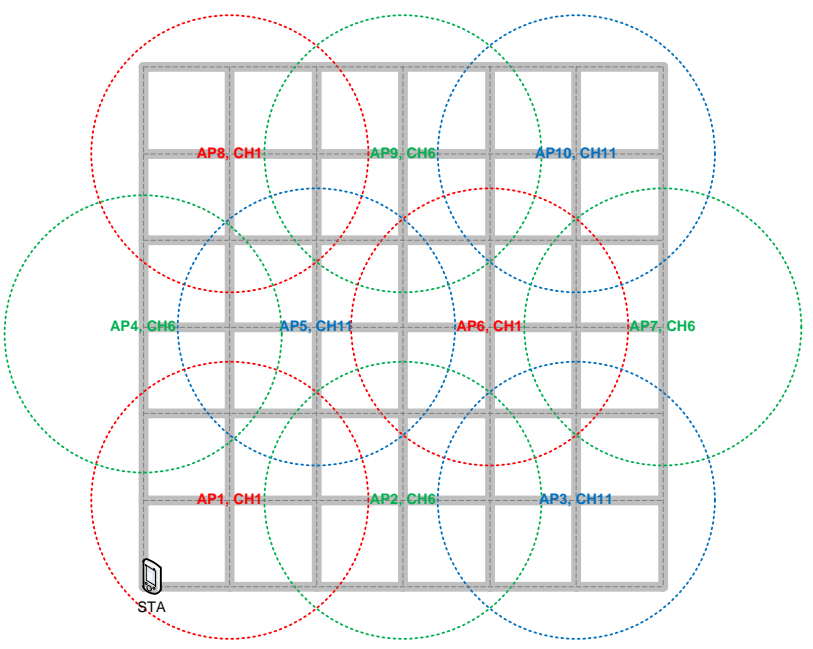

Figure 5. The deplorement of IEEE $802.11 \mathrm{~b}$ networks

TABLE II. CACHING TABLE StRUCtURE

\begin{tabular}{|c|c|c|}
\hline Current $\boldsymbol{A P}$ & Scanned AP & Connection Count \\
\hline AP1 & AP2 & 3 \\
\hline AP1 & AP3 & 1 \\
\hline AP1 & AP4 & 0 \\
\hline$\ldots$ & $\ldots$ & $\ldots$ \\
\hline APn & APm & $k$ \\
\hline
\end{tabular}




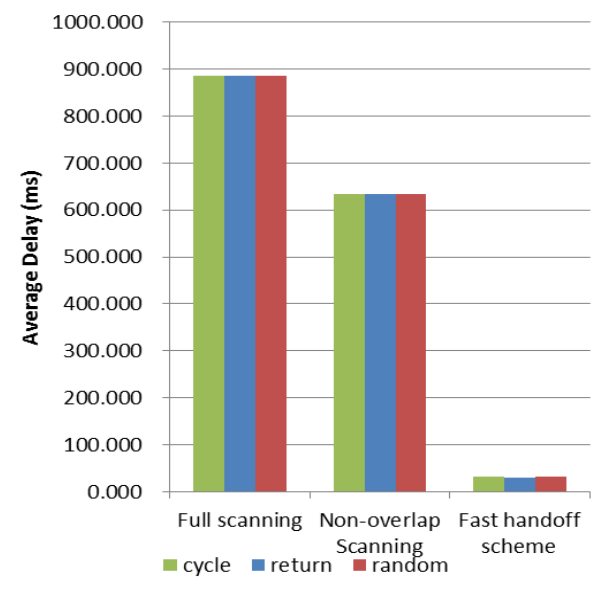

Figure 6. Anverage delay.

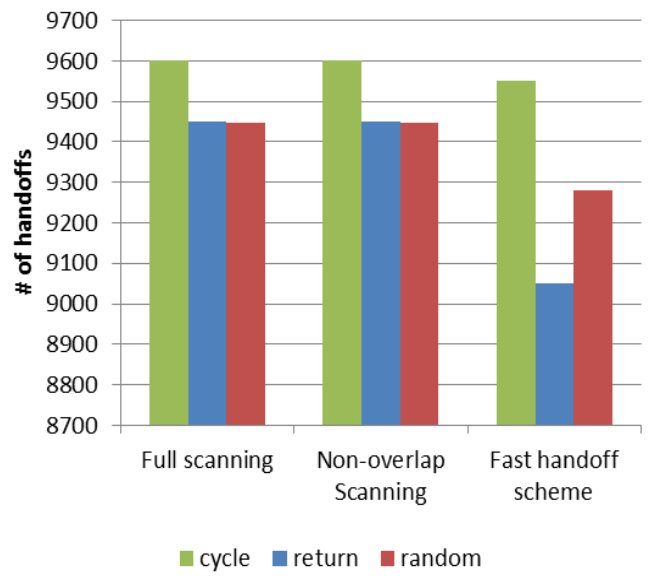

Figure 7. The number of handoffs.

\section{CONCLUTION}

This paper analyzed the delay made by handoff process, and proposed a fast handoff scheme. This fast handoff scheme is based on selective scanning and caching. It can reduce the handoff delay to about $30 \mathrm{~ms}$ and avoid necessary handoff. Simulations are implemented and the results show the proposed fast handoff scheme is useful and economical.

\section{REFERENCES}

[1] IEEE Standard 802.11. (2003). Part 11: Wireless LAN medium access control (MAC) and physical layer (PHY) specifications. ANSI/IEEE Std 802.11, 1999 edition (R2003). pp. 1-513.

[2] International Telecommunication Union. (1988). General characteristics of international telephone connections and international telephone circuits. ITU-TG.114.

[3] Mishra, A., Shin, M., \& Arbaugh, W. (2003). "An empirical analysis of the IEEE 802.11 MAC layer handoff process." ACM SIGCOMM Computer Communication Review, 33(2), 93-102.

[4] H'ector Velayos and Gunnar Karlsson, "Techniques to Reduce the IEEE 802.11b Handoff Time," in Proc. IEEE ICC'04, June 2004.

[5] Hango Purushothaman and Sumit Roy, "FastScan: a handoff scheme for voice over IEEE 802.11 WLANs", Wireless Netw (2010) 16:2049-2063.

[6] Sangho Shin, Andrea G. Forte, Anshuman Singh Rawat, and Henning Schulzrinne, "Reducing MAC layer handoff latency in IEEE 802.11 wireless LANs," In Proceedings of the second international workshop on Mobility management \& wireless access protocols (MobiWac '04). ACM, New York, NY, USA, 19-26.

[7] Sunggeun Jin; Sunghyun Choi, "A Seamless Handoff With Multiple Radios in IEEE 802.11 WLANs," Vehicular Technology, IEEE Transactions on , vol.63, no.3, pp.1408,1418, March 2014.

[8] Almulla, M.; Yikun Wang; Boukerche, A.; Zhenxia Zhang, "A fast location-based handoff scheme for vehicular networks," Communications (ICC), 2013 IEEE International Conference on , vol., no., pp.1464,1468, 9-13 June 2013.

[9] I. Shafer and M. Chang, "Movement Detection for Power-Efficient Smartphone WLAN Localization," in Proc. of the 13th ACM int'l conf. on Modeling, analysis, and simulation of wireless and mobile systems, 2010, pp. 81-90. 